\title{
A SPECTROSCOPIC SURVEY OF CATACLYSMIC VARIABLES
}

\author{
Howard E. Bond \\ Department of Physics \& Astronomy \\ Louisiana State University
}

The writer has undertaken a survey of cataclysmic variables (CV's) with the image-dissector scanner on the Kitt Peak 2.1-m reflector. A resolution of $7 \AA$ is obtained, and the wavelength interval 3500-5300 is covered. Generally only one or two observations of each object are made, with a typical integration time of $10 \mathrm{~min}$. To date, about 100 objects have been observed; most of them are classified in the GCVS as U Gem, Z Cam, or NI variables or old novae, but a number of Irregular variables (types Is, L, ?, or RCB) that appear blue on the Palomar Sky Survey prints have also been included.

Several objects were found to be misclassified as CV's, including BS Cep (an A-type star) and $\mathrm{EN}$ Cyg (type M).

One aim of this study is to establish a classification scheme for spectra of CV's. Two classification criteria are excitation and emission-line width. A wide range of excitation conditions is seen, from systems showing (In addition to the ubiquitous $H$ emission lines) Ca II emission (examples: UV Per, PY Per), through systems with He I emission (SS Aur, V425 Cas, AH Her, RU Peg, and many others), to systems with He II and C III-N III 4650 emission (CM Del, AM Her, VV Pup, V Sge, AN UMa). Line profiles range from very sharp in systems presumably seen pole-on to double-peaked in edge-on binaries. Two objects with doublepeaked emission have proven to be new eclipsing binaries: V2051 Oph $(P=90 \mathrm{~min})$ and $\mathrm{HT}$ Cas (106 min).

Typical classical novae at minimum light show continuous spectrawith very weak, high-excitation emission lines, in contrast to dwarf novae at minimum which always exhibit very strong emission lines. The state of classical novae at minimum is most similar to that of dwarf novae at maximum; that is, the primary source of light in both cases is an optically thick accretion disk. The presumed explanation is that the rate of mass transfer, $\mathrm{dM} / \mathrm{dt}$, is higher in classical novae than in dwarf novae. Classical novae do not show dwarf-nova outbursts, since they are already in states of "permanent outburst." Dwarf novae are predicted to experience classical-nova outbursts, but since the interval between outbursts is proportional to $(\mathrm{dM} / \mathrm{dt})^{-1}$, such outbursts are too rare to have been observed.

A group of CV's apparently stuck in permanent outburst includes UX UMa and CD $-42^{\circ} 14462$. KQ Mon has been discovered to be a new member of this class. They may be classical novae whose outbursts occurred in prehistoric times. 